\title{
UNIQUENESS OF STABLE MEISSNER STATE SOLUTIONS OF THE CHERN-SIMONS-HIGGS ENERGY*
}

\author{
DANIEL SPIRN ${ }^{1}$ AND XIAODONG YAN $^{2}$
}

\begin{abstract}
For external magnetic field $h_{e x} \leq C \varepsilon^{-\alpha}$, we prove that a Meissner state solution for the Chern-Simons-Higgs functional exists. Furthermore, if the solution is stable among all vortexless solutions, then it is unique.
\end{abstract}

Mathematics Subject Classification. 35J50, 49J45.

Received February 7, 2008.

Published online October 21, 2008.

\section{INTRODUCTION}

In this paper, we study uniqueness of stable Meissner solutions for the following Chern-Simons-Higgs functional

$$
G_{c s h}(u, A)=\frac{1}{2} \int_{\Omega}\left|\nabla_{A} u\right|^{2}+\frac{\mu_{\varepsilon}^{2}}{4} \frac{\left|\operatorname{curl} A-h_{e x}\right|}{|u|^{2}}+\frac{1}{\varepsilon^{2}}|u|^{2}\left(1-|u|^{2}\right)^{2} .
$$

The associated Euler-Lagrange equations for (1.1) are

$$
\begin{aligned}
-\frac{\mu_{\varepsilon}^{2}}{4} \frac{\left|\operatorname{curl} A-h_{e x}\right|^{2}}{|u|^{4}} u & =\nabla_{A}^{2} u+\frac{1}{\varepsilon^{2}} u\left(1-|u|^{2}\right)\left(3|u|^{2}-1\right) \\
0 & =-\frac{\mu_{\varepsilon}^{2}}{4} \operatorname{curl}\left(\frac{\operatorname{curl} A-h_{e x}}{|u|^{2}}\right)+j_{A}(u) .
\end{aligned}
$$

The paper is motivated by Serfaty's work [9] on Ginzburg-Landau energy where she proved uniqueness of stable Meissner state solutions for $h_{e x} \leq C \varepsilon^{-\alpha}$. In addition, it was proved in the same work that vortexless solution to Ginzburg-Landau equation continue to exists for $h_{e x}$ higher than the critical field (up to $h_{e x} \leq C \varepsilon^{-\alpha}$ ) and is locally minimizing (for $h_{e x}$ below the first critical field, it is proved by Sandier and Serfaty [8] that the vortexless solution to G-L equation is globally minimizing). The uniqueness of the Meissner state for the Ginzburg-Landau energy has been studied elsewhere, including Ye and Zhou [12] for the case with trivial gauge field and Bonnet et al. [3] for the full Ginzburg-Landau energy. In [3] the authors show uniqueness of the

Keywords and phrases. Chern-Simons-Higgs theory, superconductivity, uniqueness, Meissner solution.

* D. Spirn was supported in part by NSF grants DMS-0510121 and DMS-0707714. X. Yan was supported in part by NSF grants DMS-0700966 and DMS-0401048.

1 University of Minnesota, USA. spirn@math.umn.edu

2 University of Connecticut, USA. xiaodong@math.uconn.edu 
Meissner solution for small $\varepsilon$ and $h_{e x} \approx C \varepsilon^{-1}$ by looking for solutions in a particular function space; whereas in [9] the author showed the uniqueness of the Meissner solution for $h_{e x} \leq C \varepsilon^{-\alpha}$ for solutions in a different function space.

Remark 1.1. The study of uniqueness of solutions to the Ginzburg-Landau energy when vortices are present is much more difficult. Pacard and Riviere [7] proved uniqueness of critical points $u_{\varepsilon}$ of the Ginzburg-Landau energy with trivial gauge field when the singularities of the limiting field are nondegenerate critical points of the renormalized energy.

We follow the approach of [9] to study Meissner solutions of the Chern-Simons-Higgs energy.

Recently, the authors [10] proved existence of vortexless solutions to (1.2)-(1.3) in the case $h_{e x} \leq \frac{2|\log \varepsilon|}{\mu_{\varepsilon}^{2}}$, $1 \gg \mu_{\varepsilon} \gg \mathrm{e}^{-|\log \varepsilon|^{\alpha}}$ for $0<\alpha<1$. The solution obtained in [10] is a minimizer in

$$
V=\left\{(u, A) \in H^{1}(\Omega, \mathbb{C}) \times H^{1}\left(\Omega, \mathbb{R}^{2}\right):|u|=1 \text { on } \partial \Omega\right\} .
$$

It is also shown in [10] that for $h_{e x}$ higher than critical field, a minimizer in $V$ must have a vortex.

Remark 1.2. When $\mu_{\varepsilon} \rightarrow \mu \in(0,+\infty]$ the critical magnetic field was shown to be asymptotically $h_{c_{1}}=$ $H_{1}(\mu, \Omega)|\log \varepsilon|$, where the constant $H_{1}(\mu, \Omega)$ is calculated in terms of a scaled London equation, see $[5,6]$. A straightforward modification of the analysis of [10] shows that this critical field strength is in fact sharp and that $\left|u_{\varepsilon}\right|$ is strictly bounded away from zero.

It is a natural question to ask whether vortexless solutions continue to exist for $h_{e x}$ higher than critical field and whether it is unique. In this paper, we prove the existence of stable vortexless solutions to (1.2)-(1.3) for $h_{e x} \leq C \varepsilon^{-\alpha}$ and $\lim \sup _{\varepsilon} \mu_{\varepsilon}<\infty$. Under the additional assumption that $\mu_{\varepsilon} \geq \varepsilon^{\frac{1}{9}}$, the stable vortexless solution obtained is unique. In our setting, we define solution $(u, A)$ of $(1.2)-(1.3)$ to be vortexless if it satisfies $|u| \geq \frac{9}{10}$ in $\Omega$.

Our main results are the following theorems. We again concentrate on the technically interesting $\mu_{\varepsilon} \rightarrow 0$ case.

Theorem 1.3. There exists $\alpha_{0} \in(0,1 / 24)$ such that for $\alpha<\alpha_{0}$, if $h_{e x} \leq C \varepsilon^{-\alpha}$, and $\lim \sup _{\varepsilon} \mu_{\varepsilon}<\infty$, there exists a vortexless solution to (1.2)-(1.3) which is stable under perturbations among vortexless mappings.

Theorem 1.4. Assuming $\mu_{\varepsilon} \geq \varepsilon^{\frac{1}{9}}, \limsup _{\varepsilon} \mu_{\varepsilon}<\infty$. There exists $\alpha \in(0,1 / 24)$ and $\varepsilon_{0}$ such that, if $\varepsilon<\varepsilon_{0}$, and $h_{e x} \leq C \varepsilon^{-\alpha}$, a vortexless solution of (1.2)-(1.3) that is stable under perturbation among vortexless functions and satisfies $\int_{\Omega}|\nabla u|^{2} \leq o\left(\varepsilon^{\beta}\right)$ for some $\beta>0$ is unique. Let $E_{0}=\left\{(u, A) \in D:|u| \geq \frac{9}{10}\right\}$. For $\varepsilon<\varepsilon_{0}$, there exists a unique solution of (1.2)-(1.3) that minimizes $G_{c s h}$ over $E_{0}$, and its energy is $G_{0}+o(1)$ where

$$
G_{0}=G_{c s h}\left(1, h_{e x} \nabla^{\perp} \xi_{0}\right)
$$

and $\xi_{0}$ solves the London equation (2.1).

For $h_{e x} \leq \frac{2|\log \varepsilon|}{\mu_{\varepsilon}^{2}}, 1 \gg \mu_{\varepsilon} \gg \mathrm{e}^{-|\log \varepsilon|^{\alpha}}$ for $0<\alpha<1$, existence of solutions to (1.2)-(1.3) which satisfy $\left|u_{\varepsilon}\right| \geq \frac{1}{4}$ in $\Omega$ was obtained in [10] for all $\varepsilon<\varepsilon_{0}$. The solution obtained in [10] is a minimizer in $V$. From there it is not hard to show that $|u| \geq \frac{9}{10}$ in $\Omega$ for a smaller choice of $\varepsilon_{0}$. For $h_{e x}$ higher than the critical field (up to $\left.C \varepsilon^{-\alpha}\right)$, we will prove that vortexless solution continue to exist and is locally minimizing in $V$.

Remark 1.5. Uniqueness of periodic topological-type vortex solution has been established in the Chern-SimonsHiggs model in the self-dual case, $\mu=\varepsilon$ and $h_{e x}=0$, see $[4,11]$.

The uniqueness proof is motivated by an idea of Serfaty [9] for Ginzburg-Landau energy, $G_{g l}$ : assuming there are two solutions $\left(u_{1}, A_{1}\right)$ and $\left(u_{2}, A_{2}\right)$, she proved, through explicit computations, that

$$
G_{g l}\left(\frac{u_{1}+u_{2}}{2}, \frac{A_{1}+A_{2}}{2}\right)<\frac{G_{g l}\left(u_{1}, A_{1}\right)+G_{g l}\left(u_{2}, A_{2}\right)}{2} .
$$


It then follows that for all $t \in(0,1), G_{g l}\left((1-t) u_{1}+t u_{2},(1-t) A_{1}+t A_{2}\right) \leq \max \left(G_{g l}\left(u_{1}, A_{1}\right), G_{g l}\left(u_{2}, A_{2}\right)\right)$, which contradicts the assumed stability of solutions. The idea of Serfaty is the following: for vortexless solutions, we can write $u=\eta \mathrm{e}^{\mathrm{i} \varphi}$ and $(u, A)$ is gauge equivalent to $(\eta, A-d \varphi)=\left(\eta, A^{\prime}\right)$. The Ginzburg-Landau energy becomes

$$
G_{g l}(u, A)=\frac{1}{2} \int_{\Omega}|\eta|^{2}\left|A^{\prime}\right|^{2}+|\nabla \eta|^{2}+\frac{1}{2 \varepsilon^{2}}\left(1-\eta^{2}\right)^{2}+\left|d A^{\prime}-h_{e x}\right|^{2} .
$$

The term $I(\eta)=\int_{\Omega} \frac{1}{2 \varepsilon^{2}}\left(1-\eta^{2}\right)^{2}$ is convex for vortexless solutions $\left(\eta \geq \frac{3}{4}\right)$; it follows that

$$
\frac{I\left(\eta_{1}\right)+I\left(\eta_{2}\right)}{2}-I\left(\frac{\eta_{1}+\eta_{2}}{2}\right) \geq \frac{C}{\varepsilon^{2}} \int_{\Omega}\left(\eta_{1}-\eta_{2}\right)^{2}
$$

On the other hand for $K\left(\eta, A^{\prime}\right)=\int_{\Omega}|\eta|^{2}\left|A^{\prime}\right|^{2}$, direct calculation shows

$$
\left|\frac{K\left(\eta_{1}, A_{1}^{\prime}\right)+K\left(\eta_{2}, A_{2}^{\prime}\right)}{2}-K\left(\frac{\eta_{1}+\eta_{2}}{2}, \frac{A_{1}^{\prime}+A_{2}^{\prime}}{2}\right)\right| \leq C\left(\max \left(\left|A_{1}^{\prime}\right|_{L^{\infty}},\left|A_{2}^{\prime}\right|_{L^{\infty}}\right)\right)^{2} \int_{\Omega}\left(\eta_{1}-\eta_{2}\right)^{2} .
$$

Since $\left|A_{i}^{\prime}\right|_{L^{\infty}}=o\left(\frac{1}{\varepsilon}\right)$, the convex term from $\int_{\Omega} \frac{1}{2 \varepsilon^{2}}\left(1-\eta^{2}\right)^{2}$ dominates over $\int_{\Omega}|\eta|^{2}|A|^{2}$ and (1.4) follows from (1.5), (1.6) and the convexity of the rest of the terms.

In our case, under the same gauge choice, the Chern-Simons-Higgs energy becomes

$$
G_{c s h}(u, A)=\frac{1}{2} \int_{\Omega} \eta^{2}\left|A^{\prime}\right|^{2}+|\nabla \eta|^{2}+\frac{1}{\varepsilon^{2}} \eta^{2}\left(1-\eta^{2}\right)^{2}+\frac{\mu_{\varepsilon}^{2}}{4} \frac{\left|\operatorname{curl} A^{\prime}-h_{e x}\right|^{2}}{\eta^{2}}
$$

The term $\int_{\Omega} \frac{1}{\varepsilon^{2}} \eta^{2}\left(1-\eta^{2}\right)^{2}$ is convex for vortexless solutions $\left(\eta \geq \frac{9}{10}\right)$ with a similar bound from below as (1.5) and the term $\int_{\Omega} \eta^{2}\left|A^{\prime}\right|^{2}$ is controlled above by (1.6). Finally for term $L\left(\eta, A^{\prime}\right)=\int_{\Omega} \frac{\mu_{\varepsilon}^{2}}{4} \frac{\left|\operatorname{curl} A^{\prime}-h_{e x}\right|^{2}}{\eta^{2}}$, we have

$$
\left|\frac{L\left(\eta_{1}, A_{1}^{\prime}\right)+L\left(\eta_{2}, A_{2}^{\prime}\right)}{2}-L\left(\frac{\eta_{1}+\eta_{2}}{2}, \frac{A_{1}^{\prime}+A_{2}^{\prime}}{2}\right)\right| \leq C\left(\max \left(\left|\operatorname{curl} A_{1}^{\prime}\right|_{L^{\infty}},\left|\operatorname{curl} A_{2}^{\prime}\right|_{L^{\infty}}\right)\right)^{2} \int_{\Omega}\left(\eta_{1}-\eta_{2}\right)^{2} .
$$

Since $\left|A^{\prime}\right|_{L^{\infty}}=o\left(\frac{1}{\varepsilon}\right),\left|\operatorname{curl} A^{\prime}\right|_{L^{\infty}}=o\left(\frac{1}{\varepsilon}\right)$ (Lem. 3.3), we obtain the same conclusion.

\section{Proof of existence}

Following [10], we introduce the following notation.

$$
F(u)=\frac{1}{2} \int_{\Omega}|\nabla u|^{2}+\frac{1}{\varepsilon^{2}}|u|^{2}\left(1-|u|^{2}\right)^{2}
$$

and we assume

$$
A=d^{*} \xi, \quad \xi=h_{e x} \xi_{0}+\zeta
$$

where

$$
\left\{\begin{array}{cc}
-\frac{\mu_{\varepsilon}^{2}}{4} \Delta^{2} \xi_{0}+\Delta \xi_{0}=0 & \text { in } \Omega \\
\Delta \xi_{0}=1 & \text { on } \partial \Omega \\
\xi_{0}=0 & \text { on } \partial \Omega
\end{array}\right.
$$

and

$$
\zeta=\Delta \zeta=0 \quad \text { on } \partial \Omega
$$

We quote the following estimate from [10]. 
Lemma 2.1. Suppose $|\Omega| \leq F, G_{c s h}(u, A) \leq M_{\varepsilon}$ and $\eta=|u| \geq \frac{1}{2}$ on $\partial \Omega$, then for all $2<p<\infty$ and $0<\beta<\frac{2}{p}$, the following estimates hold

$$
\begin{gathered}
\|\eta\|_{H^{1}} \leq C \sqrt{M_{\varepsilon}}, \\
\left\|1-\eta^{2}\right\|_{L^{2}} \leq C \varepsilon M_{\varepsilon}, \\
\|1-\eta\|_{L^{p}} \leq C_{p, \beta} \varepsilon^{\beta} M_{\varepsilon}^{\frac{1+\beta}{2}}, \\
\|\eta\|_{L^{p}} \leq C_{p, \beta} \varepsilon^{\beta} M_{\varepsilon}^{\frac{1+\beta}{2}}+|\Omega| .
\end{gathered}
$$

Moreover, for all $1 \leq \alpha<2,0<\beta<\frac{2-\alpha}{\alpha}$, we have bounds

$$
\begin{gathered}
\left\|j_{A}(u)\right\|_{L^{\alpha}} \leq\left(C_{\alpha, \beta} \varepsilon^{\beta} M_{\varepsilon}^{\frac{1+\beta}{2}}+|\Omega|\right) M_{\varepsilon}^{\frac{1}{2}}, \\
\left\|h-h_{e x}\right\|_{L^{\alpha}} \leq \frac{C_{\alpha, \beta}}{\mu_{\varepsilon}} \sqrt{M_{\varepsilon}}\left(C_{\alpha, \beta} \varepsilon^{\beta} M_{\varepsilon}^{\frac{1+\beta}{2}}+|\Omega|\right),
\end{gathered}
$$

where $C_{\alpha, \beta} \longrightarrow \infty$ as $\alpha \longrightarrow 2$. If $(u, A)$ is a weak solution of (1.3), we have

$$
\left\|\frac{h-h_{e x}}{\eta^{2}}\right\|_{W^{1, q}} \leq \frac{C_{q}}{\mu_{\varepsilon}^{2}} \sqrt{M_{\varepsilon}}\left(C_{q, \beta} \varepsilon^{\beta} M_{\varepsilon}^{\frac{1+\beta}{2}}+|\Omega|\right)
$$

for all $1 \leq q<2,0<\beta<\frac{2-q}{q}$.

An immediate corollary of Lemma 2.1 is the following lemma.

Lemma 2.2. Given $h_{e x} \leq C \varepsilon^{-\alpha}$ for some $0<\alpha<\frac{1}{24}$, $\lim \sup \mu_{\varepsilon}<\infty$. If $G_{c s h}(u, A) \leq M_{\varepsilon}=C \mu_{\varepsilon}^{2} h_{e x}^{2}$, then for any $2<p<\infty$,

$$
\|\eta\|_{L^{p}} \leq C_{p} M_{\varepsilon}+|\Omega|
$$

if $2<p<22$,

$$
\|\eta\|_{L^{p}} \leq C_{p}
$$

Moreover, if (u,A) satisfies (1.3), $A=d^{*} \xi$, there exists $\beta>0$, such that

$$
|\nabla \xi|_{L^{\infty}} \leq \frac{C}{\mu_{\varepsilon}^{2}} \sqrt{M_{\varepsilon}}\left(C_{\beta} \varepsilon^{\beta} M_{\varepsilon}^{\frac{1+\beta}{2}}+|\Omega|\right)+C h_{e x} ;
$$

in particular, this implies

$$
|\nabla \xi|_{L^{\infty}} \leq \frac{C}{\mu_{\varepsilon}^{2}} M_{\varepsilon}^{\frac{3}{2}}+C h_{e x}
$$

Proof. (2.9) follows directly from (2.5). By (2.5), we have

$$
\|\eta\|_{L^{p}} \leq C \varepsilon^{\beta} \mu_{\varepsilon}^{1+\beta} \varepsilon^{-\alpha(1+\beta)}+|\Omega|,
$$

pick $\beta$ close to $\frac{2}{p}$, for $0<\alpha<\frac{1}{24}$, $\lim \sup \mu_{\varepsilon}<\infty$, (2.10) follows from (2.13) when $2<p<22$. To prove (2.11), since

$$
\left\|h-h_{e x}\right\|_{L^{r}} \leq\left\|\frac{h-h_{e x}}{\eta^{2}}\right\|_{L^{t}}\|\eta\|_{L^{2 s}}^{2}
$$


with $\frac{1}{r}=\frac{1}{t}+\frac{1}{s}$. Pick $2<r<s<11$, there exists $q<2$ such that $\frac{2 q}{2-q}>t=\frac{r s}{s-r}$. By (2.8) and Sobolev embedding, we deduce

$$
\begin{aligned}
\left\|\frac{h-h_{e x}}{\eta^{2}}\right\|_{L^{t}} & \leq C\left\|\frac{h-h_{e x}}{\eta^{2}}\right\|_{W^{1, q}} \\
& \leq \frac{C_{q}}{\mu_{\varepsilon}^{2}} \sqrt{M_{\varepsilon}}\left(C_{q, \beta} \varepsilon^{\beta} M_{\varepsilon}^{\frac{1+\beta}{2}}+|\Omega|\right)
\end{aligned}
$$

for $0<\beta<\frac{2-q}{q}$. (2.11) follows from (2.10), (2.14), (2.15) and Sobolev embedding. Finally (2.12) follows directly from $(2.11)$.

Following idea of proof of Lemma 2.3 in [10], applying estimates in Lemmas 2.1 and 2.2, we have the following gradient estimate.

Lemma 2.3. Assume $(u, A)$ is a solution of (1.2)-(1.3) satisfying $\frac{\partial u}{\partial \nu}=0$ on $\partial \Omega$ and $G_{c s h}(u, A) \leq M_{\varepsilon}$, $h_{e x} \leq \frac{\sqrt{M_{\varepsilon}}}{\mu_{\varepsilon}}$. If $\varepsilon \frac{M_{\varepsilon}^{2}}{\mu_{\varepsilon}} \leq C$, we have

$$
|\nabla u| \leq \frac{C_{0}}{\varepsilon}
$$

where $C_{0}$ is a constant independent of $u, A$, and $\varepsilon, \mu_{\varepsilon}$

We introduce the following regularization of $u$ (similar regularization for Ginzburg-Landau energy is introduced in [1] and used in [9]). Given any $0<\gamma<1$, for any $(u, A) \in V, u^{\gamma}$ is defined as a minimizer for

$$
\inf _{\substack{H^{1}(\Omega, \mathbb{C}) \\|v|=1 \text { on } \partial \Omega}} \frac{1}{2} \int_{\Omega}|\nabla v|^{2}+\frac{1}{\varepsilon^{2}}|v|^{2}\left(1-|v|^{2}\right)^{2}+\frac{|v-u|^{2}}{\varepsilon^{2 \gamma}} .
$$

Lemma 2.4. $u^{\gamma}$ is in $H^{3}(\Omega, \mathbb{C})$ and satisfies

$$
\begin{aligned}
-\Delta u^{\gamma} & =\frac{1}{\varepsilon^{2}} u^{\gamma}\left(1-\left|u^{\gamma}\right|^{2}\right)\left(3\left|u^{\gamma}\right|^{2}-1\right)+\frac{u-u^{\gamma}}{\varepsilon^{2 \gamma}} \\
F\left(u^{\gamma}\right) & \leq F(u) \\
\left|\nabla u^{\gamma}\right| & \leq \frac{C}{\varepsilon} .
\end{aligned}
$$

Proof. Follow the same proof as in [1,2], where we replace $\frac{1}{\varepsilon^{2}} u^{\gamma}\left(1-\left|u^{\gamma}\right|^{2}\right)$ with $\frac{1}{\varepsilon^{2}} u^{\gamma}\left(1-\left|u^{\gamma}\right|^{2}\right)\left(3\left|u^{\gamma}\right|^{2}-1\right)$.

Since $\left|\nabla u^{\gamma}\right| \leq \frac{C}{\varepsilon}$, the vortices of $u^{\gamma}$ are well defined. The following ball construction lemma is a variation of the ball construction used in [10].

Proposition 2.5. There exists $\alpha \in(0,1 / 24)$, such that if $h_{e x} \leq C \varepsilon^{-\alpha}$, let $u: \Omega \longrightarrow \mathbb{C}$ be such that $|\nabla u|_{\infty} \leq \frac{C_{0}}{\varepsilon}$, $|u|=1$ on $\partial \Omega$ and $F(u) \leq C \varepsilon^{-2 \alpha}$. Then there exist disjoint balls $\left\{B_{i}\right\}_{i \in I}$ such that for sufficiently small $\varepsilon$

(1) $\left\{|u(x)|<\frac{10}{11}\right\} \subset \cup_{i} B_{i}$.

(2) $\operatorname{card} I \leq C \varepsilon^{-2 \alpha}$.

(3) $r_{i} \leq C \frac{\varepsilon^{\frac{1}{2}}}{|\log \varepsilon|}$.

(4) If $\overline{B_{i}} \subset \Omega$, and $d_{i}=\operatorname{deg}\left(u, \partial B_{i}\right)$, then

$$
F\left(u, B_{i}\right) \geq \pi \frac{\left|d_{i}\right|}{3}|\log \varepsilon|-C .
$$


Proof. Follow the proof of Proposition 2.13 in [10], choosing $s_{1}=\varepsilon^{\frac{2}{3}}$ in the initial step, replacing the assumption $h_{e x} \leq C \frac{|\log \varepsilon|}{\mu_{\varepsilon}^{2}}$ by $h_{e x} \leq C \varepsilon^{-\alpha}$ and $\frac{1}{2}$ by $\frac{10}{11}$.

We recall the definitions

$$
\begin{aligned}
& V(\xi)=\frac{1}{2} \int|\nabla \xi|^{2}+|\Delta \xi|^{2}+2 \pi \sum_{i \in I} d_{i} \xi\left(a_{i}\right)-h_{e x} \int_{\Omega} \Delta \xi \\
& \widetilde{V}(\zeta)=\frac{1}{2} \int_{\Omega}|\nabla \zeta|^{2}+|\Delta \zeta|^{2}+2 \pi \sum_{i \in I} d_{i} \zeta\left(a_{i}\right) .
\end{aligned}
$$

Lemma 2.6. There exists $\alpha \in(0,1 / 24)$ such that if $h_{e x} \leq C \varepsilon^{-\alpha}$, $\lim _{\sup _{\varepsilon \rightarrow 0}} \mu_{\varepsilon}<\infty$, given $(u, A)$ satisfying (1.3) and $F(u) \leq C \mu_{\varepsilon}^{2} h_{e x}^{2}$, the energy can be split as

$$
\begin{aligned}
G_{c s h}(u, A) & =F(u)+V(\xi)+o\left(\varepsilon^{\beta}\right) \\
& =G_{0}+F(u)+2 \pi h_{e x} \sum_{i \in I} d_{i} \xi_{0}\left(a_{i}\right)+\widetilde{V}(\zeta)+o\left(\varepsilon^{\beta}\right),
\end{aligned}
$$

where $\left(a_{i}, d_{i}\right)$ denote the vortices of $u^{\gamma} . G_{0}=\int_{\Omega} \frac{h_{e x}^{2}}{2}\left|\nabla \xi_{0}\right|^{2}+\frac{\mu_{\varepsilon}^{2}}{8} h_{e x}^{2}\left|\Delta \xi_{0}-1\right|^{2}, \beta=\beta(\alpha)>0$.

Proof. Write

$$
\begin{gathered}
\left|\nabla_{A} u\right|^{2}=|\nabla u|^{2}+|\nabla \xi|^{2}+\left(1-\eta^{2}\right)|\nabla \xi|^{2}+2\left(\mathrm{i} u, \xi_{x_{2}} u_{x_{1}}-\xi_{x_{1}} u_{x_{2}}\right), \\
\left|\frac{h-h_{e x}}{\eta}\right|^{2}=\left|h-h_{e x}\right|^{2}+\frac{\left|h-h_{e x}\right|^{2}}{|u|^{4}}|u|^{2}\left(1-|u|^{2}\right) .
\end{gathered}
$$

Since $(u, A)$ satisfies (1.3), by (2.3) and (2.12), we conclude

$$
\begin{aligned}
\int_{\Omega}\left(1-\eta^{2}\right)|\nabla \xi|^{2} & \leq C|\nabla \xi|_{L^{\infty}}^{2}\left\|1-\eta^{2}\right\|_{L^{2}} \\
& \leq C\left(\frac{M_{\varepsilon}^{\frac{3}{2}}}{\mu_{\varepsilon}^{2}}+h_{e x}\right)^{2} \varepsilon M_{\varepsilon} \\
& \leq C\left(\mu_{\varepsilon} h_{e x}^{3}+h_{e x}\right)^{2} \varepsilon \mu_{\varepsilon}^{2} h_{e x}^{2} \\
& \leq C \varepsilon^{1-8 \alpha},
\end{aligned}
$$

and for $\frac{1}{p}+\frac{1}{q}=\frac{1}{2}$, by $(2.3),(2.9)$ and $(2.15)$

$$
\begin{aligned}
\int_{\Omega} \frac{\left|h-h_{e x}\right|^{2}}{|u|^{4}}|u|^{2}\left(1-|u|^{2}\right) & \leq\left\|\frac{\left|h-h_{e x}\right|}{|u|^{2}}\right\|_{L^{2 p}}^{2}\|\eta\|_{L^{2 q}}^{2}\left\|1-\eta^{2}\right\|_{L^{2}} \\
& \leq C\left(\frac{M_{\varepsilon}^{\frac{3}{2}}}{\mu_{\varepsilon}^{2}}\right)^{2} M_{\varepsilon}^{2} \varepsilon M_{\varepsilon} \\
& \leq C \varepsilon^{1-12 \alpha} .
\end{aligned}
$$

Therefore

$$
G_{c s h}(u, A)=\frac{1}{2} \int_{\Omega}|\nabla u|^{2}+|\nabla \xi|^{2}+\frac{\mu_{\varepsilon}^{2}}{4}\left|h-h_{e x}\right|^{2}+2\left(\mathrm{i} u, \xi_{x_{2}} u_{x_{1}}-\xi_{x_{1}} u_{x_{2}}\right)+o\left(\varepsilon^{\beta}\right) .
$$


The rest of the proof follows from similar argument as in Lemmas 4.2 and 4.3 in [9], replacing the assumption $F(u)<M|\log \varepsilon|$ and $h_{e x} \leq C|\log \varepsilon|$ by $F(u) \leq C \mu_{\varepsilon}^{2} h_{e x}^{2}, h_{e x} \leq C \varepsilon^{-\alpha}$.

Lemma 2.7. Let $\alpha, h_{e x}$ and $\mu_{\varepsilon}$ satisfy the same assumptions as in Lemma 2.6. If (u,A) is a solution of (1.2)(1.3) such that $u^{\gamma}$ has no vortex $\left(\left|u^{\gamma}\right| \geq \frac{9}{10}\right)$ and that $G_{c s h}(u, A) \leq G_{0}$ and $F(u) \leq C \mu_{\varepsilon}^{2} h_{e x}^{2}$, then $u$ has no vortex in $\Omega$.

Proof. From Lemma 2.6 and the assumption, we obtain

$$
G_{0} \geq G_{c s h}(u, A)=G_{0}+F(u)+\widetilde{V}(\zeta)+o\left(\varepsilon^{\beta}\right),
$$

therefore

$$
F(u)+\widetilde{V}(\zeta) \leq o\left(\varepsilon^{\beta}\right)
$$

Since $(u, A)$ is a solution of (1.2)-(1.3), by elliptic estimates (Lem. 2.3), we have $|\nabla u| \leq \frac{C}{\varepsilon}$. Therefore the vortex structure of $u$ is well defined and (2.17) implies $u$ is vortexless.

Proposition 2.8. There exists $\alpha \in(0,1 / 24)$ and $\varepsilon_{0}$ such that if $\varepsilon<\varepsilon_{0}$ and $h_{e x} \leq C \varepsilon^{-\alpha}, \limsup _{\varepsilon} \mu_{\varepsilon}<\infty$, there exists a solution $(u, A)$ of (1.2)-(1.3) satisfying $|u| \geq \frac{9}{10}$, that is a local minimizer of $J$ in $V$. In addition,

$$
\inf _{\theta \in[0,2 \pi]}\left\|(u, \xi)-\left(\mathrm{e}^{\mathrm{i} \theta}, h_{e x} \xi_{0}\right)\right\| \longrightarrow 0 \quad \text { as } \quad \varepsilon \longrightarrow 0
$$

where

$$
\|(u, z)\|^{2}=\|\nabla u\|_{L^{2}}^{2}+\|u\|_{L^{2}}^{2}+\|\nabla z\|_{L^{2}}^{2}+\|\Delta z\|_{L^{2}}^{2}
$$

Proof. Let

$$
G_{k}(u, A)=\frac{1}{2} \int_{\Omega}\left|\nabla_{A} u\right|^{2}+\frac{\mu_{\varepsilon}^{2}}{4} \frac{\left|\operatorname{curl} A-h_{e x}\right|}{|u|^{2}+\frac{1}{k^{2}}}+\frac{1}{\varepsilon^{2}}|u|^{2}\left(1-|u|^{2}\right)^{2} .
$$

Consider the open domain

$$
U=\left\{(u, A) \in V: F(u)+\frac{1}{2} \int_{\Omega}|\nabla \zeta|^{2}+|\Delta \zeta|^{2}<\varepsilon^{\frac{\beta}{2}}\right\}
$$

where $\beta$ is given by Lemma 2.6. There exists $\left(v_{k}, A_{k}\right) \in \bar{U}$ which achieves $\min _{\bar{U}} G_{k}$ and $\left(v_{k}, A_{k}\right)$ satisfies

$$
0=-\frac{\mu_{\varepsilon}^{2}}{4} \operatorname{curl}\left(\frac{\operatorname{curl} A_{k}-h_{e x}}{\left|v_{k}\right|^{2}+\frac{1}{k^{2}}}\right)+j_{A_{k}}\left(v_{k}\right) .
$$

This can be shown by the following argument. Given $\left(u_{k}^{n}, A_{k}^{n}\right)$ minimizing sequence of $G_{k}$, since

$$
\begin{gathered}
F\left(u_{k}^{n}\right)+\frac{1}{2} \int_{\Omega}\left|\nabla \zeta_{k}^{n}\right|^{2}+\left|\Delta \zeta_{k}^{n}\right|^{2} \leq \varepsilon^{\frac{\beta}{2}}, \\
A_{k}^{n}=h_{e x} d^{*} \xi_{0}+d^{*} \zeta
\end{gathered}
$$

we conclude $\left(u_{k}^{n}, A_{k}^{n}\right)$ is a bounded sequence in $H^{1}(\Omega, \mathbb{C}) \times H^{1}\left(\Omega, \mathbb{R}^{2}\right)$. Subject to a subsequence, we can assume $\left(u_{k}^{n}, A_{k}^{n}\right) \rightarrow\left(v_{k}, A_{k}\right)$ in $H^{1}(\Omega, \mathbb{C}) \times H^{1}\left(\Omega, \mathbb{R}^{2}\right)$ as $n \longrightarrow \infty$ and

$$
\begin{aligned}
G_{k}\left(v_{k}, A_{k}\right) & \leq \lim \inf _{n \rightarrow \infty} G_{k}\left(u_{k}^{n}, A_{k}^{n}\right) \\
F\left(v_{k}\right)+\frac{1}{2} \int_{\Omega}\left|\nabla \zeta_{k}\right|^{2}+\left|\Delta \zeta_{k}\right|^{2} & \leq \lim \inf _{n \rightarrow \infty} F\left(u_{k}^{n}\right)+\frac{1}{2} \int_{\Omega}\left|\nabla \zeta_{k}^{n}\right|^{2}+\left|\Delta \zeta_{k}^{n}\right|^{2} .
\end{aligned}
$$


Therefore $\left(v_{k}, A_{k}\right)$ is a minimizer of $G_{k}$ in $\bar{U}$. Applying Lemma 2.4 and Proposition 2.5 to $v_{k}$, we obtain

$$
\begin{aligned}
\varepsilon^{\frac{\beta}{2}} & >F\left(v_{k}\right) \geq F\left(v_{k}^{\gamma}\right) \\
& \geq \pi \sum_{i \in L} \frac{\left|d_{i}\right|}{3}|\log \varepsilon|-C,
\end{aligned}
$$

where $L$ is the collection of vortex balls for $v_{k}^{\gamma}$. This implies $L=\emptyset$, i.e. $v_{k}^{\gamma}$ has no vortex $\left(\right.$ since $\left.d_{i} \neq 0\right)$. Moreover, when $\frac{1}{k^{2}}<\varepsilon$, we can prove a similar energy splitting formula for $G_{k}$ as Lemma 2.6,

$$
G_{k}\left(v_{k}, A_{k}\right)=G_{0}+F\left(v_{k}\right)+\frac{1}{2} \int_{\Omega}\left|\nabla \zeta_{k}\right|^{2}+\left|\Delta \zeta_{k}\right|^{2}+o\left(\varepsilon^{\beta}\right)
$$

On the other hand, $\left(1, h_{e x} \nabla^{\perp} \xi_{0}\right) \in U$ is a comparison map, by minimality of $\left(v_{k}, A_{k}\right)$, we obtain $G_{c s h}\left(v_{k}, A_{k}\right) \leq$ $G_{0}$. This together with (2.20) implies

$$
F\left(v_{k}\right)+\frac{1}{2} \int_{\Omega}\left|\nabla \zeta_{k}\right|^{2}+\left|\Delta \zeta_{k}\right|^{2} \leq o\left(\varepsilon^{\beta}\right) .
$$

This guarantees $\left(v_{k}, A_{k}\right) \in \stackrel{\circ}{U}$, i.e. $\left(v_{k}, A_{k}\right)$ is a local minimizer of $G_{k}$ and satisfies

$$
\begin{aligned}
-\frac{\mu_{\varepsilon}^{2}}{4} \frac{\left|\operatorname{curl} A_{k}-h_{e x}\right|^{2}}{\left(\left|v_{k}\right|^{2}+\frac{1}{k^{2}}\right)^{2}} u & =\nabla_{A}^{2} v_{k}+\frac{1}{\varepsilon^{2}} v_{k}\left(1-\left|v_{k}\right|^{2}\right)\left(3\left|v_{k}\right|^{2}-1\right) \\
0 & =-\frac{\mu^{2}}{4} \operatorname{curl}\left(\frac{\operatorname{curl} A_{k}-h_{e x}}{\left|v_{k}\right|^{2}+\frac{1}{k^{2}}}\right)+j_{A_{k}}\left(v_{k}\right) .
\end{aligned}
$$

By elliptic estimates (similar to Lem. 2.1), $\left(v_{k}, A_{k}\right)$ is bounded in $H^{1} \times H^{1}$. Up to a subsequence, we assume $\left(v_{k}, A_{k}\right) \rightarrow(u, A)$ in $H^{1} \times H^{1}$ where $(u, A)$ satisfies $(1.2)-(1.3)$ and

$$
G_{c s h}(u, A) \leq \lim \inf _{k \longrightarrow \infty} G_{k}\left(v_{k}, A_{k}\right) .
$$

Given a minimizing sequence $\left(u_{k}, B_{k}\right)$ of $G_{c s h}$ in $U$, we have

$$
G_{c s h}\left(u_{k}, B_{k}\right) \geq G_{k}\left(u_{k}, B_{k}\right) \geq G_{k}\left(v_{k}, A_{k}\right) .
$$

(2.23) implies $(u, A)$ is a minimizer of $G_{c s h}$ in $U$ and $(u, A) \in \bar{U}$. We repeat the regularization argument for $u$ and conclude $u^{\gamma}$ is vortexless. By Lemma 2.7, $u$ is vortexless. Finally, since $|u|=1$ on $\partial \Omega$, energy estimates imply $\left\|1-|u|^{2}\right\|_{L^{2}} \leq o(1)$, from here (2.18) can be proved following exact same argument of step 2 in the proof of Proposition 3.1 in [9].

\section{Proof of Uniqueness}

We assume that $h_{e x} \leq C \varepsilon^{-\alpha}$ and $\mu_{\varepsilon} \geq \varepsilon^{\frac{1}{9}}$. We prove that if a Meissner solution $(u, A)$ exists and stable under perturbation among vortexless mappings, then it is unique among the solutions satisfying $\|\nabla u\|_{L^{2}}^{2} \leq o\left(\varepsilon^{\beta}\right)$. (Here $\beta$ is given by Lem. 2.6.) In particular, a solution $(u, A)$ that is minimizing among all vortexless solutions is unique.

We prove uniqueness by contradiction. If there are two distinct stable solutions $\left(u_{1}, A_{1}\right)$ and $\left(u_{2}, A_{2}\right)$ of $(1.2)$ and (1.3) with $\operatorname{div} A_{j}=0, A_{j} \cdot \nu=0$ on $\partial \Omega$ and $\left\|\nabla u_{j}\right\|_{L^{2}}^{2} \leq o\left(\varepsilon^{\beta}\right)$. We assume $G_{c s h}\left(u_{1}, A_{1}\right) \leq G_{c s h}\left(u_{2}, A_{2}\right)$. Denote $\eta_{j}=\left|u_{j}\right|$. 
Lemma 3.1. For $j=1,2,\left(u_{j}, A_{j}\right)$ is gauge equivalent to $\left(\eta_{j}, B_{j}\right)$ with

$$
\begin{gathered}
\operatorname{div}\left(\eta_{j}^{2} B_{j}\right)=0 \\
G_{c s h}\left(u_{j}, A_{j}\right)=\frac{1}{2} \int_{\Omega} \eta_{j}^{2} B_{j}^{2}+\left|\nabla \eta_{j}\right|^{2}+\frac{1}{\varepsilon^{2}} \eta_{j}^{2}\left(1-\eta_{j}^{2}\right)^{2}+\frac{\mu_{\varepsilon}^{2}}{4} \frac{\left|\operatorname{curl} B_{j}-h_{e x}\right|^{2}}{\eta_{j}^{2}} .
\end{gathered}
$$

Proof. Since $\eta_{j} \geq \frac{9}{10}$, we can write $u_{j}=\eta_{j} \mathrm{e}^{\mathrm{i} \phi_{j}}$ globally on $\Omega$. We write $B_{j}=A_{j}-\nabla \phi_{j}$, then $\left(u_{j}, A_{j}\right)$ is gauge equivalent to

$$
\left(u_{j} \mathrm{e}^{-i \phi_{j}}, A_{j}-\nabla \phi_{j}\right)=\left(\eta_{j}, B_{j}\right)
$$

and $\operatorname{curl} A_{j}=\operatorname{curl} B_{j}$. Since $\int_{\Omega}\left|\nabla_{A} u\right|^{2}$ is invariant under gauge-transformations,

$$
\int_{\Omega}\left|\nabla_{A_{j}} u_{j}\right|^{2}=\int_{\Omega}\left|\nabla_{B_{j}} \eta_{j}\right|^{2}=\int_{\Omega}\left|\nabla \eta_{j}-\mathrm{i} B_{j} \eta_{j}\right|^{2}=\int_{\Omega} \eta_{j}^{2} B_{j}^{2}+\left|\nabla \eta_{j}\right|^{2} .
$$

The expression (3.2) follows. For (3.1), notice that equation (1.3) gives

$$
-\frac{\mu_{\varepsilon}^{2}}{4} \operatorname{curl}\left(\frac{\operatorname{curl} A_{j}-h_{e x}}{\left|u_{j}\right|^{2}}\right)=\left(\mathrm{i} u_{j}, \nabla_{A_{j}} u_{j}\right)=\left(\mathrm{i} \eta_{j}, \nabla_{B_{j}} \eta_{j}\right)=-\eta_{j}^{2} B_{j},
$$

take divergence on both sides, we get $\operatorname{div}\left(\eta_{j}^{2} B_{j}\right)=0$.

A direct corollary of Lemmas 2.1 and 2.3 is the following

Lemma 3.2. If $(u, A)$ is weak solution of (1.2)-(1.3) satisfying $\frac{\partial u}{\partial \nu}=0$ on $\partial \Omega$, the following holds for any $1<q \leq 4, \frac{3}{4} \leq \delta<1$,

$$
\begin{gathered}
\left\|j_{A}(u)\right\|_{L^{q}(\Omega)} \leq \frac{C_{q}}{\mu_{\varepsilon}^{2}} M_{\varepsilon}^{\frac{3}{2}}+C h_{e x}+\frac{C(\Omega, \delta)}{\varepsilon^{\delta}}\left(\sqrt{M_{\varepsilon}}\right)^{1-\delta} \\
\left\|\frac{h-h_{e x}}{\eta^{2}}\right\|_{W^{1, q}(\Omega)} \leq\left(\frac{C_{q}}{\mu_{\varepsilon}^{2}} M_{\varepsilon}^{\frac{3}{2}}+C h_{e x}+\frac{C(\Omega, \delta)}{\varepsilon^{\delta}}\left(\sqrt{M_{\varepsilon}}\right)^{1-\delta}\right) \frac{1}{\mu_{\varepsilon}^{2}} .
\end{gathered}
$$

In particular, this implies

$$
\|\operatorname{curl} A\|_{L^{\infty}(\Omega)} \leq\left(\frac{C_{q}}{\mu_{\varepsilon}^{2}} M_{\varepsilon}^{\frac{3}{2}}+C h_{e x}+\frac{C(\Omega, \delta)}{\varepsilon^{\delta}}\left(\sqrt{M_{\varepsilon}}\right)^{1-\delta}\right) \frac{1}{\mu_{\varepsilon}^{2}} .
$$

Proof. Since $j_{A}(u)=\left(\mathrm{i} u, \nabla_{A} u\right)=(\mathrm{i} u, \nabla u-\mathrm{i} A u)$, it follows from $(2.5),(2.12)$ and Lemma 2.3 that for $1<q \leq 4$,

$$
\begin{aligned}
\left\|j_{A}(u)\right\|_{L^{q}(\Omega)} & \leq\|\nabla u\|_{L^{\infty}(\Omega)}^{\delta}\left\||u||\nabla u|^{1-\delta}\right\|_{L^{q}(\Omega)}+\|A\|_{L^{\infty}(\Omega)}\left\||u|^{2}\right\|_{L^{q}} \\
& \leq \frac{C_{q}}{\mu_{\varepsilon}^{2}} M_{\varepsilon}^{\frac{3}{2}}+C h_{e x}+\frac{C(\Omega, \delta)}{\varepsilon^{\delta}}\left(\sqrt{M_{\varepsilon}}\right)^{1-\delta} .
\end{aligned}
$$

(3.4) follows from elliptic estimates for equations (1.3) and (3.3). Finally (3.5) follows from (2.10), (3.4) and Sobolev embedding.

Lemma 3.3. Given $\left(u_{j}, A_{j}\right)$ stable Meissner state solution and satisfying $\left\|\nabla u_{j}\right\|_{L^{2}}^{2} \leq o\left(\varepsilon^{\beta}\right)$, $\beta$ is given by Lemma 2.6. If $G_{c s h}\left(u_{j}, A_{j}\right) \leq C \mu_{\varepsilon}^{2} h_{e x}^{2}, h_{e x} \leq C \varepsilon^{-\alpha}, 0<\alpha<\frac{1}{24}$ and $\mu_{\varepsilon} \geq \varepsilon^{\frac{1}{9}}$, $\lim \sup \mu_{\varepsilon}<\infty$, then as $\varepsilon \longrightarrow 0$,

$$
\begin{gathered}
\left\|B_{j}\right\|_{L^{\infty}(\Omega)} \leq o\left(\frac{1}{\varepsilon}\right) \\
\left\|\operatorname{curl} B_{j}\right\|_{L^{\infty}(\Omega)} \leq o\left(\frac{1}{\varepsilon}\right) .
\end{gathered}
$$


Proof. We follow idea of [9] to prove (3.6). If we assume $\left(u_{j}, A_{j}\right)$ is energy minimizing among vortexless solutions, then

$$
G_{c s h}\left(u_{j}, A_{j}\right) \leq G_{c s h}\left(1, h_{e x} \nabla^{\perp} \xi_{0}\right)=G_{0} \leq C \mu_{\varepsilon}^{2} h_{e x}^{2} .
$$

Decomposing $\xi=h_{e x} \xi_{0}+\zeta$ and dropping the subscript $j$, we obtain

$$
\begin{aligned}
G_{0} \geq & G_{c s h}(u, A) \\
\geq & \frac{1}{2} \int_{\Omega}|\nabla u|^{2}+|\nabla \xi|^{2} \\
& +\frac{\mu_{\varepsilon}^{2}}{4} \frac{\left|\Delta \xi-h_{e x}\right|^{2}}{|u|^{2}}+\frac{1}{\varepsilon^{2}}|u|^{2}\left(1-|u|^{2}\right)^{2} \\
& +o\left(\varepsilon^{\beta}\right) \\
= & G_{0}+F(u)+\frac{1}{2} \int_{\Omega}|\Delta \zeta|^{2}+|\nabla \zeta|^{2}+o\left(\varepsilon^{\beta}\right) .
\end{aligned}
$$

Therefore

$$
\int_{\Omega}|\nabla u|^{2}=\int_{\Omega}|\nabla \eta|^{2}+\eta^{2}|\nabla \phi|^{2} \leq o\left(\varepsilon^{\beta}\right)
$$

for some $\beta>0$. We now assume this condition is satisfied. From Lemma 2.1, we have $\left\|A_{j}\right\|_{L^{\infty}} \leq \frac{C_{q}}{\mu^{2}} M_{\varepsilon}^{\frac{3}{2}}+C h_{e x}$. Therefore

$$
\begin{aligned}
\left\|B_{j}\right\|_{L^{\infty}} & \leq\left\|A_{j}\right\|_{L^{\infty}}+\|\nabla \phi\|_{L^{\infty}} \\
& \leq \frac{C}{\varepsilon} .
\end{aligned}
$$

For any $p>1$, by interpolation, we have

$$
\begin{aligned}
\|\nabla \eta\|_{L^{p}} & \leq C\|\nabla \eta\|_{L^{\infty}}^{1-\frac{2}{p}}\|\nabla \eta\|_{L^{2}}^{\frac{2}{p}} \\
& \leq C \varepsilon^{-1+\frac{2}{p}} \varepsilon^{\frac{\beta}{p}} \\
& \leq C \varepsilon^{\gamma}
\end{aligned}
$$

for some $\gamma>0$, provided $p<\beta+2$. On the other hand, from (3.1), we have

$$
\eta^{2} \operatorname{div} B_{j}=-2 \eta \nabla \eta \cdot B_{j}
$$

which implies

We deduce that

$$
-\Delta \phi=-\frac{2}{\eta} \nabla \eta \cdot B_{j}
$$

Choosing $2<p<\beta+2$, we have

$$
\|\Delta \phi\|_{L^{p}} \leq C\left\|B_{j}\right\|_{L^{\infty}}\|\nabla \eta\|_{L^{p}}
$$

$$
\|\Delta \phi\|_{L^{p}} \leq C \frac{\varepsilon^{\gamma}}{\varepsilon} \leq o\left(\frac{1}{\varepsilon}\right) .
$$

Since $\frac{\partial u}{\partial \nu}=0$ implies $\frac{\partial \phi}{\partial \nu}=0$ on $\partial \Omega$. From elliptic estimates and Sobolev embedding we deduce that

$$
\|\nabla \phi\|_{L^{\infty}} \leq o\left(\frac{1}{\varepsilon}\right)
$$


from which follows

$$
\left\|B_{j}\right\|_{L^{\infty}} \leq o\left(\frac{1}{\varepsilon}\right) .
$$

Finally since $\operatorname{curl} B_{j}=\operatorname{curl} A_{j}$, if $\mu_{\varepsilon} \geq \varepsilon^{\frac{1}{9}}$, taking $\delta=\frac{3}{4}$ in (3.5), (3.7) follows directly.

We are going to prove that

$$
\begin{aligned}
G_{c s h}\left(\frac{\eta_{1}+\eta_{2}}{2}, \frac{B_{1}+B_{2}}{2}\right) & <\frac{G_{c s h}\left(\eta_{1}, B_{1}\right)+G_{c s h}\left(\eta_{2}, B_{2}\right)}{2} \\
& \leq G_{c s h}\left(\eta_{2}, B_{2}\right)
\end{aligned}
$$

thus getting a contradiction to the assumption that $\left(u_{2}, A_{2}\right)$ is stable.

Lemma 3.4. If $\left(\eta_{1}, B_{1}\right) \neq\left(\eta_{2}, B_{2}\right)$, then

$$
\begin{aligned}
& \int_{\Omega}\left(\frac{\eta_{1}+\eta_{2}}{2}\right)^{2}\left|\frac{B_{1}+B_{2}}{2}\right|^{2}+\frac{\mu_{\varepsilon}^{2}}{4}\left|\frac{\operatorname{curl} \frac{B_{1}+B_{2}}{2}-h_{e x}}{\frac{\eta_{1}+\eta_{2}}{2}}\right|^{2}+\int_{\Omega} \frac{1}{\varepsilon^{2}}\left(\frac{\eta_{1}+\eta_{2}}{2}\right)^{2}\left(1-\left(\frac{\eta_{1}+\eta_{2}}{2}\right)^{2}\right)^{2} \leq \\
& \frac{1}{2} \int_{\Omega} \eta_{1}^{2}\left|B_{1}\right|^{2}+\frac{\mu_{\varepsilon}^{2}}{4}\left|\frac{\operatorname{curl} B_{1}-h_{e x}}{\eta_{1}}\right|^{2}+\frac{1}{\varepsilon^{2}} \eta_{1}^{2}\left(1-\eta_{1}^{2}\right)^{2}+\frac{1}{2} \int_{\Omega} \eta_{2}^{2}\left|B_{2}\right|^{2}+\frac{\mu_{\varepsilon}^{2}}{4}\left|\frac{\operatorname{curl} B_{2}-h_{e x}}{\eta_{2}}\right|^{2}+\frac{1}{\varepsilon^{2}} \eta_{2}^{2}\left(1-\eta_{2}^{2}\right)^{2} .
\end{aligned}
$$

Proof. We compute $X=X_{1}+X_{2}+X_{3}$, where

$$
\begin{gathered}
X_{1}=\frac{1}{2} \int_{\Omega} \eta_{1}^{2}\left|B_{1}\right|^{2}+\eta_{2}^{2}\left|B_{2}\right|^{2}-\int_{\Omega}\left(\frac{\eta_{1}+\eta_{2}}{2}\right)^{2}\left|\frac{B_{1}+B_{2}}{2}\right|^{2}, \\
X_{2}=\frac{1}{2} \int_{\Omega} \frac{1}{\varepsilon^{2}} \eta_{1}^{2}\left(1-\eta_{1}^{2}\right)^{2}+\frac{1}{\varepsilon^{2}} \eta_{2}^{2}\left(1-\eta_{2}^{2}\right)^{2}-\int_{\Omega} \frac{1}{\varepsilon^{2}}\left(\frac{\eta_{1}+\eta_{2}}{2}\right)^{2}\left(1-\left(\frac{\eta_{1}+\eta_{2}}{2}\right)^{2}\right)^{2} \\
X_{3}=\frac{1}{2} \int_{\Omega} \frac{\mu_{\varepsilon}^{2}}{4}\left|\frac{\operatorname{curl} B_{1}-h_{e x}}{\eta_{1}}\right|^{2}+\frac{\mu_{\varepsilon}^{2}}{4}\left|\frac{\operatorname{curl} B_{2}-h_{e x}}{\eta_{2}}\right|^{2}-\int_{\Omega} \frac{\mu_{\varepsilon}^{2}}{4}\left|\frac{\operatorname{curl} \frac{B_{1}+B_{2}}{2}-h_{e x}}{\frac{\eta_{1}+\eta_{2}}{2}}\right|^{2} .
\end{gathered}
$$

Following [9], we have

$$
\begin{aligned}
X_{1}= & \frac{1}{16} \int_{\Omega}\left(\eta_{1}-\eta_{2}\right)^{2}\left|B_{1}+B_{2}\right|^{2}+4 \eta_{1}^{2}\left|B_{1}-B_{2}\right|^{2} \\
& +\left(\eta_{2}-\eta_{1}\right)\left(B_{2}-B_{1}\right) \cdot\left(B_{1}\left(-2 \eta_{1}-4 \eta_{2}\right)+B_{2}\left(-6 \eta_{1}-8 \eta_{2}\right)\right) .
\end{aligned}
$$

Since $u_{1}, u_{2}$ are vortexless solutions, we know that $\frac{9}{10} \leq \eta_{j}$ for $j=1,2$. This guarantees $\eta_{1}, \eta_{2}$ lie in the domain of convexity of function $f(x)=x^{2}\left(1-x^{2}\right)^{2}$. In particular, when $x_{1}, x_{2} \geq \frac{9}{10}$, through Taylor expansion, 
we have (assuming $\left.x_{1} \leq x_{2}\right)$

$$
\begin{aligned}
\frac{1}{2}\left(f\left(x_{1}\right)+f\left(x_{2}\right)\right)-f\left(\frac{x_{1}+x_{2}}{2}\right)= & \frac{1}{2}\left(f\left(x_{1}\right)-f\left(\frac{x_{1}+x_{2}}{2}\right)\right)+\frac{1}{2}\left(f\left(x_{2}\right)-f\left(\frac{x_{1}+x_{2}}{2}\right)\right) \\
= & \frac{1}{2}\left[f^{\prime}\left(\frac{x_{1}+x_{2}}{2}\right)\left(x_{1}-\frac{x_{1}+x_{2}}{2}\right)+f^{\prime \prime}\left(\widetilde{x_{1}}\right)\left(\frac{x_{1}-x_{2}}{2}\right)^{2}\right] \\
& +\frac{1}{2}\left[f^{\prime}\left(\frac{x_{1}+x_{2}}{2}\right)\left(x_{2}-\frac{x_{1}+x_{2}}{2}\right)+f^{\prime \prime}\left(\widetilde{x_{2}}\right)\left(\frac{x_{1}-x_{2}}{2}\right)^{2}\right] \\
& =\frac{1}{2}\left(f^{\prime \prime}\left(\widetilde{x_{1}}\right)+f^{\prime \prime}\left(\widetilde{x_{2}}\right)\right)\left(\frac{x_{1}-x_{2}}{2}\right)^{2} \\
\geq & 2 \cdot\left(\frac{x_{1}-x_{2}}{2}\right)^{2} .
\end{aligned}
$$

Here $\widetilde{x_{1}} \in\left(x_{1}, \frac{x_{1}+x_{2}}{2}\right)$ and $\widetilde{x_{2}} \in\left(\frac{x_{1}+x_{2}}{2}, x_{2}\right)$ satisfying $\widetilde{x_{1}}, \widetilde{x_{2}} \geq \frac{9}{10}$, in the last step, we used this and the fact that $f^{\prime \prime}\left(\widetilde{x}_{i}\right) \geq f^{\prime \prime}\left(\frac{9}{10}\right) \geq 2$. From $(3.13)$, we obtain estimates for $X_{2}$ :

$$
\begin{aligned}
X_{2}= & \frac{1}{2 \varepsilon^{2}} \int_{\Omega}\left[\eta_{1}^{2}\left(1-\eta_{1}^{2}\right)^{2}-\left(\frac{\eta_{1}+\eta_{2}}{2}\right)^{2}\left(1-\left(\frac{\eta_{1}+\eta_{2}}{2}\right)^{2}\right)^{2}\right] \\
& +\frac{1}{2 \varepsilon^{2}} \int_{\Omega}\left[\eta_{2}^{2}\left(1-\eta_{2}^{2}\right)^{2}-\left(\frac{\eta_{1}+\eta_{2}}{2}\right)^{2}\left(1-\left(\frac{\eta_{1}+\eta_{2}}{2}\right)^{2}\right)^{2}\right] \\
\geq & \frac{1}{\varepsilon^{2}} \int_{\Omega} 2 \cdot\left(\frac{\eta_{1}-\eta_{2}}{2}\right)^{2}=\frac{1}{2 \varepsilon^{2}} \int_{\Omega}\left(\eta_{1}-\eta_{2}\right)^{2} .
\end{aligned}
$$

For $X_{3}$, we denote $y_{j}=\frac{\mu_{\varepsilon}}{2}\left(\operatorname{curl} B_{j}-h_{e x}\right), j=1,2$. Then

$$
\begin{aligned}
X_{3}= & \frac{1}{2} \int_{\Omega}\left(\frac{y_{1}}{\eta_{1}}\right)^{2}+\left(\frac{y_{2}}{\eta_{2}}\right)^{2}-2\left(\frac{y_{1}+y_{2}}{\eta_{1}+\eta_{2}}\right)^{2} \\
= & \frac{1}{2} \int_{\Omega}\left(\frac{y_{1}}{\eta_{1}}+\frac{y_{1}+y_{2}}{\eta_{1}+\eta_{2}}\right)\left(\frac{y_{1}}{\eta_{1}}-\frac{y_{1}+y_{2}}{\eta_{1}+\eta_{2}}\right) \\
& +\frac{1}{2} \int_{\Omega}\left(\frac{y_{2}}{\eta_{2}}+\frac{y_{1}+y_{2}}{\eta_{1}+\eta_{2}}\right)\left(\frac{y_{2}}{\eta_{2}}-\frac{y_{1}+y_{2}}{\eta_{1}+\eta_{2}}\right) \\
= & \frac{1}{2} \int_{\Omega} \frac{y_{1} \eta_{2}-y_{2} \eta_{1}}{\eta_{1}+\eta_{2}}\left(\frac{y_{1}}{\eta_{1}^{2}}-\frac{y_{2}}{\eta_{2}^{2}}+\frac{y_{1}+y_{2}}{\eta_{1}+\eta_{2}}\left(\frac{1}{\eta_{1}}-\frac{1}{\eta_{2}}\right)\right) \\
= & \frac{1}{2} \int_{\Omega} \frac{y_{1}\left(\eta_{2}-\eta_{1}\right)+\left(y_{1}-y_{2}\right) \eta_{1}}{\eta_{1}+\eta_{2}} \cdot \frac{y_{1}\left(\eta_{2}^{2}-\eta_{1}^{2}\right)+\left(y_{1}-y_{2}\right) \eta_{1}^{2}}{\eta_{1}^{2} \eta_{2}^{2}} \\
& +\frac{1}{2} \int_{\Omega} \frac{y_{1}\left(\eta_{2}-\eta_{1}\right)+\left(y_{1}-y_{2}\right)+\eta_{1}}{\left.\eta_{2}\right)^{2}} \cdot\left(y_{1}+y_{2}\right) \cdot \frac{\left(\eta_{2}-\eta_{1}\right)}{\eta_{1} \eta_{2}} \\
= & \frac{1}{2} \int_{\Omega} \frac{y_{1}^{2}\left(\eta_{2}-\eta_{1}\right)^{2}}{\eta_{1}^{2} \eta_{2}^{2}}+\frac{y_{1} \cdot\left(\eta_{2}-\eta_{1}\right)\left(y_{1}-y_{2}\right)}{\eta_{2}^{2}\left(\eta_{1}+\eta_{2}\right)}+\frac{y_{1} \cdot\left(\eta_{2}-\eta_{1}\right)\left(y_{1}-y_{2}\right)}{\eta_{1} \eta_{2}^{2}} \\
& +\frac{1}{2} \int_{\Omega} \frac{y_{1}\left(y_{1}+y_{2}\right)\left(\eta_{2}-\eta_{1}\right)^{2}}{\left(\eta_{1}+\eta_{2}\right)^{2} \eta_{1} \eta_{2}}+\frac{\left(y_{1}+y_{2}\right)\left(\eta_{2}-\eta_{1}\right)\left(y_{1}-y_{2}\right)}{\eta_{2}\left(\eta_{1}+\eta_{2}\right)^{2}}+\frac{\left(y_{1}-y_{2}\right)^{2} \eta_{1}}{\eta_{2}^{2}\left(\eta_{1}+\eta_{2}\right)}
\end{aligned}
$$


Note the integrand in $X_{3}$ is symmetric in indices 1,2 , we deduce

$$
\begin{aligned}
X_{3}= & \frac{1}{2} \int_{\Omega} \frac{y_{2}^{2}\left(\eta_{2}-\eta_{1}\right)^{2}}{\eta_{1}^{2} \eta_{2}^{2}}+\frac{y_{2} \cdot\left(\eta_{2}-\eta_{1}\right)\left(y_{1}-y_{2}\right)}{\eta_{1}^{2}\left(\eta_{1}+\eta_{2}\right)}+\frac{y_{2} \cdot\left(\eta_{2}-\eta_{1}\right)\left(y_{1}-y_{2}\right)}{\eta_{2} \eta_{1}^{2}} \\
& +\frac{1}{2} \int_{\Omega} \frac{y_{2}\left(y_{1}+y_{2}\right)\left(\eta_{2}-\eta_{1}\right)^{2}}{\left(\eta_{1}+\eta_{2}\right)^{2} \eta_{1} \eta_{2}}+\frac{\left(y_{1}+y_{2}\right)\left(\eta_{2}-\eta_{1}\right)\left(y_{1}-y_{2}\right)}{\eta_{1}\left(\eta_{1}+\eta_{2}\right)^{2}}+\frac{\left(y_{1}-y_{2}\right)^{2} \eta_{2}}{\eta_{1}^{2}\left(\eta_{1}+\eta_{2}\right)}
\end{aligned}
$$

Therefore

$$
\begin{aligned}
X_{3}= & \frac{1}{4} \int_{\Omega} \frac{\left(y_{1}^{2}+y_{2}^{2}\right)\left(\eta_{2}-\eta_{1}\right)^{2}}{\eta_{1}^{2} \eta_{2}^{2}}+\frac{\left(y_{1}+y_{2}\right)^{2}\left(\eta_{2}-\eta_{1}\right)^{2}}{\left(\eta_{1}+\eta_{2}\right)^{2} \eta_{1} \eta_{2}}+\frac{1}{4} \int_{\Omega} \frac{\left(y_{1}-y_{2}\right)^{2}}{\left(\eta_{1}+\eta_{2}\right)}\left(\frac{\eta_{1}}{\eta_{2}^{2}}+\frac{\eta_{2}}{\eta_{1}^{2}}\right) \\
& +\frac{1}{4} \int_{\Omega}\left(\eta_{2}-\eta_{1}\right)\left(y_{1}-y_{2}\right)\left[\frac{y_{2}}{\eta_{1}^{2}\left(\eta_{1}+\eta_{2}\right)}+\frac{y_{1}}{\eta_{2}^{2}\left(\eta_{1}+\eta_{2}\right)}\right. \\
& \left.+\frac{y_{2}}{\eta_{2} \eta_{1}^{2}}+\frac{y_{1}}{\eta_{1} \eta_{2}^{2}}+\frac{\left(y_{1}+y_{2}\right)}{\eta_{1}\left(\eta_{1}+\eta_{2}\right)^{2}}+\frac{\left(y_{1}+y_{2}\right)}{\eta_{2}\left(\eta_{1}+\eta_{2}\right)^{2}}\right]
\end{aligned}
$$

By (3.7),

$$
\left\|y_{j}\right\|_{L^{\infty}} \leq\left\|\operatorname{curl} B_{j}\right\|_{L^{\infty}}+h_{e x} \leq o\left(\frac{1}{\varepsilon}\right) .
$$

If we assume for contradiction that $X \leq 0$, combining (3.12), (3.14) and (3.15) we obtain

$$
\begin{aligned}
& \frac{1}{4} \int_{\Omega} \frac{\left(y_{1}^{2}+y_{2}^{2}\right)\left(\eta_{2}-\eta_{1}\right)^{2}}{\eta_{1}^{2} \eta_{2}^{2}}+\frac{\left(y_{1}+y_{2}\right)^{2}\left(\eta_{2}-\eta_{1}\right)^{2}}{\left(\eta_{1}+\eta_{2}\right)^{2} \eta_{1} \eta_{2}}+\frac{1}{4} \int_{\Omega} \frac{\left(y_{1}-y_{2}\right)^{2}}{\left(\eta_{1}+\eta_{2}\right)}\left(\frac{\eta_{1}}{\eta_{2}^{2}}+\frac{\eta_{2}}{\eta_{1}^{2}}\right) \\
& +\frac{1}{2 \varepsilon^{2}} \int_{\Omega}\left(\eta_{2}-\eta_{1}\right)^{2}+\frac{1}{16} \int_{\Omega}\left(\eta_{1}-\eta_{2}\right)^{2}\left|B_{1}+B_{2}\right|^{2}+4 \eta_{1}^{2}\left|B_{1}-B_{2}\right|^{2} \leq \\
& C\left\|\eta_{1}-\eta_{2}\right\|_{L^{2}}\left\|B_{1}-B_{2}\right\|_{L^{2}}\left(\left\|B_{1}\right\|_{L^{\infty}}+\left\|B_{2}\right\|_{L^{\infty}}\right)+C\left\|\eta_{1}-\eta_{2}\right\|_{L^{2}}\left\|y_{1}-y_{2}\right\|_{L^{2}}\left(\left\|y_{1}\right\|_{L^{\infty}}+\left\|y_{2}\right\|_{L^{\infty}}\right) .
\end{aligned}
$$

We remark here that in the first term of the last inequality, we used the boundedness of $\eta_{i}$. In fact, taking $p=4$ and $\beta$ close to $\frac{1}{2}$ in $(2.4)$, we conclude

$$
\left\|1-\eta_{i}\right\|_{L^{4}} \leq C
$$

From here and (3.8), boundedness of $\eta_{i}$ follows from Sobolev embedding. On the other hand,

$$
\begin{aligned}
& \frac{1}{4} \int_{\Omega} \frac{\left(y_{1}^{2}+y_{2}^{2}\right)\left(\eta_{2}-\eta_{1}\right)^{2}}{\eta_{1}^{2} \eta_{2}^{2}}+\frac{\left(y_{1}+y_{2}\right)^{2}\left(\eta_{2}-\eta_{1}\right)^{2}}{\left(\eta_{1}+\eta_{2}\right)^{2} \eta_{1} \eta_{2}}+\frac{1}{4} \int_{\Omega} \frac{\left(y_{1}-y_{2}\right)^{2}}{\left(\eta_{1}+\eta_{2}\right)}\left(\frac{\eta_{1}}{\eta_{2}^{2}}+\frac{\eta_{2}}{\eta_{1}^{2}}\right)+\frac{1}{2 \varepsilon^{2}} \int_{\Omega}\left(\eta_{2}-\eta_{1}\right)^{2} \\
& +\frac{1}{16} \int_{\Omega}\left(\eta_{1}-\eta_{2}\right)^{2}\left|B_{1}+B_{2}\right|^{2}+4 \eta_{1}^{2}\left|B_{1}-B_{2}\right|^{2} \geq \\
& \frac{C}{\varepsilon}\left(\left\|\eta_{1}-\eta_{2}\right\|_{L^{2}}\left\|B_{1}-B_{2}\right\|_{L^{2}}+\left\|\eta_{1}-\eta_{2}\right\|_{L^{2}}\left\|y_{1}-y_{2}\right\|_{L^{2}}\right) .
\end{aligned}
$$

Since $\left\|y_{j}\right\|_{L^{\infty}} \leq o\left(\frac{1}{\varepsilon}\right),\left\|B_{j}\right\|_{L^{\infty}} \leq o\left(\frac{1}{\varepsilon}\right)$, we must have $\eta_{1}=\eta_{2}$ or $B_{1}=B_{2}$. If $\eta_{1}=\eta_{2}$, simple convexity argument gives

$$
\begin{aligned}
\int_{\Omega}\left(\eta_{1}\right)^{2}\left|\frac{B_{1}+B_{2}}{2}\right|^{2}+\frac{\mu_{\varepsilon}^{2}}{4}\left|\frac{\operatorname{curl} \frac{B_{1}+B_{2}}{2}-h_{e x}}{\eta_{1}}\right|^{2}< & \frac{1}{2} \int_{\Omega} \eta_{1}^{2}\left|B_{1}\right|^{2}+\frac{\mu_{\varepsilon}^{2}}{4}\left|\frac{\operatorname{curl} B_{1}-h_{e x}}{\eta_{1}}\right|^{2} \\
& +\frac{1}{2} \int_{\Omega} \eta_{2}^{2}\left|B_{2}\right|^{2}+\frac{\mu_{\varepsilon}^{2}}{4}\left|\frac{\operatorname{curl} B_{2}-h_{e x}}{\eta_{2}}\right|^{2}
\end{aligned}
$$


thus $X>0\left(\right.$ since $\left.B_{1} \neq B_{2}\right)$. If $B_{1}=B_{2}$, again by convexity (since $\left.\eta_{i} \geq \frac{9}{10}\right)$

$$
\begin{aligned}
& \int_{\Omega}\left(\frac{\eta_{1}+\eta_{2}}{2}\right)^{2}\left|B_{1}\right|^{2}+\frac{\mu_{\varepsilon}^{2}}{4}\left|\frac{\operatorname{curl} B_{1}-h_{e x}}{\frac{\eta_{1}+\eta_{2}}{2}}\right|^{2}+\frac{1}{\varepsilon^{2}}\left(\frac{\eta_{1}+\eta_{2}}{2}\right)^{2}\left(1-\left(\frac{\eta_{1}+\eta_{2}}{2}\right)^{2}\right)^{2} \leq \\
& \frac{1}{2} \int_{\Omega} \eta_{1}^{2}\left|B_{1}\right|^{2}+\frac{\mu_{\varepsilon}^{2}}{4}\left|\frac{\operatorname{curl} B_{1}-h_{e x}}{\eta_{1}}\right|^{2}+\frac{1}{\varepsilon^{2}} \eta_{1}^{2}\left(1-\eta_{1}^{2}\right)^{2}+\frac{1}{2} \int_{\Omega} \eta_{2}^{2}\left|B_{2}\right|^{2}+\frac{\mu_{\varepsilon}^{2}}{4}\left|\frac{\operatorname{curl} B_{2}-h_{e x}}{\eta_{2}}\right|^{2}+\frac{1}{\varepsilon^{2}} \eta_{2}^{2}\left(1-\eta_{2}^{2}\right)^{2}
\end{aligned}
$$

and $X>0$ (since $\eta_{1} \neq \eta_{2}$ ). We are led to contradiction in all cases therefore $X>0$ and lemma is proved.

Lemma 3.5. If $\mu_{\varepsilon} \geq \varepsilon^{\frac{1}{9}}$ and $\limsup \mu_{\varepsilon}<\infty$, there exists $\alpha \in(0,1 / 24)$ and $\varepsilon_{0}$ such that, if $\varepsilon<\varepsilon_{0}$, a stable vortexless solution of (1.2)-(1.3) for $h_{e x} \leq C \varepsilon^{-\alpha}$ with $\int_{\Omega}|\nabla u|^{2} \leq o\left(\varepsilon^{\beta}\right)$ for some $\beta>0$ is unique. Let $E_{0}=\left\{(u, A) \in D:|u| \geq \frac{9}{10}\right\}$. For $\varepsilon<\varepsilon_{0}$, if there exists a solution of (1.2)-(1.3) that minimizes $G_{c s h}$ over $E_{0}$, then it is unique.

Proof. Lemma 3.4 implies

$$
\begin{aligned}
G_{c s h}\left(\frac{\eta_{1}+\eta_{2}}{2}, \frac{B_{1}+B_{2}}{2}\right) & <\frac{G_{c s h}\left(\eta_{1}, B_{1}\right)+G_{c s h}\left(\eta_{2}, B_{2}\right)}{2} \\
& \leq G_{c s h}\left(\eta_{2}, B_{2}\right) .
\end{aligned}
$$

A standard argument gives

$$
G_{c s h}\left((1-t) \eta_{1}+t \eta_{2},(1-t) B_{1}+t B_{2}\right)<G_{c s h}\left(\eta_{2}, B_{2}\right)
$$

for all $t \in(0,1)$, this contradicts the stability of $\left(\eta_{2}, B_{2}\right)$. Hence $\eta_{1}=\eta_{2}, B_{1}=B_{2}$.

\section{REFERENCES}

[1] L. Almeida and F. Bethuel, Topological methods for the Ginzburg-Landau equations. J. Math. Pures. Appl. 77 (1998) 1-49.

[2] F. Bethuel, H. Brezis and F. Hélein, Asymptotics for the minimization of a Ginzburg-Landau functional. Cal. Var. Partial Differ. Equ. 1 (1993) 123-148.

[3] A. Bonnet, S.J. Chapman and R. Monneau, Convergence of Meissner minimizers of the Ginzburg-Landau energy of superconductivity as $\kappa \rightarrow+\infty$. SIAM J. Math. Anal. 31 (2000) 1374-1395.

[4] K. Choe and H.-S. Nam, Existence and uniqueness of topological multivortex solutions of the self-dual Chern-Simons $C P(1)$ model. Nonlinear Anal. 66 (2007) 2794-2813.

[5] M. Kurzke and D. Spirn, Gamma limit of the nonself-dual Chern-Simons-Higgs energy. J. Funct. Anal. 244 (2008) 535-588.

[6] M. Kurzke and D. Spirn, Scaling limits of the Chern-Simons-Higgs energy. Commun. Contemp. Math. 10 (2008) 1-16.

[7] F. Pacard and T. Rivière, Linear and nonlinear aspects of vortices. The Ginzburg-Landau model. Progress in Nonlinear Differential Equations and their Applications 39. Birkhäuser Boston, Inc., Boston, MA, USA (2000).

[8] E. Sandier and S. Serfaty, Global minimizers for the Ginzburg-Landau functional below the first critical magnetic field. Ann. Inst. H. Poincaré, Anal. Non Linéaire 17 (2000) 119-145.

[9] S. Serfaty, Stable configurations in superconductivity: Uniqueness, mulitplicity, and vortex-nucleation. Arch. Rational Mech. Anal. 149 (1999) 329-365.

[10] D. Spirn and X. Yan, Minimizers near the first critical field for the nonself-dual Chern-Simons-Higgs energy. Calc. Var. Partial Differ. Equ. (to appear).

[11] G. Tarantello, Uniqueness of selfdual periodic Chern-Simons vortices of topological-type. Calc. Var. Partial Differ. Equ. 29 (2007) 191-217.

[12] D. Ye and F. Zhou, Uniqueness of solutions of the Ginzburg-Landau problem. Nonlinear Anal. 26 (1996) 603-612. 